\title{
On the Majorana Equation: Relations between Its Complex Two-Component and Real Four-Component Eigenfunctions
}

\author{
Eckart Marsch \\ Institute for Experimental and Applied Physics, Christian Albrechts University at Kiel, \\ Leibnizstraße 11, 24118 Kiel, Germany \\ Correspondence should be addressed to Eckart Marsch, marsch@physik.uni-kiel.de
}

Received 2 July 2012; Accepted 24 July 2012

Academic Editors: K. Ammari, A. Herrera-Aguilar, D. Ida, and A. Sanyal

Copyright (C) 2012 Eckart Marsch. This is an open access article distributed under the Creative Commons Attribution License, which permits unrestricted use, distribution, and reproduction in any medium, provided the original work is properly cited.

\begin{abstract}
We first derive without recourse to the Dirac equation the two-component Majorana equation with a mass term by a direct linearization of the relativistic dispersion relation of a massive particle. Thereby, we make only use of the complex conjugation operator and the Pauli spin matrices, corresponding to the irreducible representation of the Lorentz group. Then we derive the complex two-component eigenfunctions of the Majorana equation and the related quantum fields in a concise way, by exploiting the so-called chirality conjugation operator that involves the spin-flip operator. Subsequently, the four-component spinor solutions of the real Majorana equation are derived, and their intrinsic relations with the spinors of the complex two-component version of the Majorana equation are revealed and discussed extensively.
\end{abstract}

\section{Introduction}

In this paper we first present a new derivation [1] of the two-component Majorana equation including a mass term by a direct linearization of the relativistic dispersion relation of a massive particle, in a way similar to that used originally by Dirac [2]. We subsequently derive from the two-component complex the four-component real version of the Majorana equation [3]. We thus obtain some new results and look at the problem from a fresh theoretical perspective, thus expanding the established theoretical framework as described in modern monographs $[4,5]$ on this subject. One intention of this work is to obtain the Majorana equation "completely on its own rather than as an afterthought when treating the Dirac equation," as it was phrased by Case [6], who first reformulated half a century ago the Majorana theory of the neutrino. Another main objective is to address the key question of whether Majorana particles are their own antiparticles, and they are according to common 
textbook wisdom. We will show that a real four-component or complex two-component spinor wavefunction does not imply this conclusion necessarily, but following Mannheim [7] we will consistently define antiparticles as being associated with the negative root of the dispersion relation and particles with the positive one.

The recent paper by Pal [8] titled "Dirac, Majorana, and Weyl fermions" discussed the definitions of and connections between these three fields and their intrinsic symmetries. We consider the present paper partly as a complement to his largely tutorial work, yet here with emphasis on the two-component Majorana equation for a massive fermion, which is treated without recourse to the Dirac equation [1]. Thereby we only make use of the complex conjugation operator and the Pauli spin matrices, corresponding to the irreducible representation of the Lorentz group. The Lorentz-invariant complex conjugation [8] involves the here introduced spin-flip operator. Its connection to chiral symmetry is also discussed. Finally, we also show that Dirac's equation in the real Majorana representation is a direct consequence of the two-component complex Majorana equation as obtained in the following.

We derive in much detail the eigenfunctions of the Majorana equation and the related quantum fields, yet other than in the paper by Case [6] in a direct way, whereby we will exploit the spin-flip operator, or as we also may call it perhaps more adequately, the chirality conjugation operator. Pal [8] gave in the introduction to his paper a good motivation, which we fully agree with, for dealing again with this theoretical subject. Our main motivation is to contribute to the ongoing discussion of whether massive neutrinos are Dirac or Majorana fermions and to better understand the latter theoretically in terms of the two-component theory and its connection to the four-component real version. In this respect we also complement the old pedagogical review on Majorana masses by Mannheim [7].

\section{New Derivation of the Majorana Equation and Its Symmetries}

In this section a new derivation of the two-component Majorana equation is presented, by linearizing the standard dispersion relation of a massive relativistic particle. Following Dirac [2] historically, one can derive his equation in a straightforward way from the relativistic energy and momentum relation, $E=\sqrt{m^{2}+\mathbf{p}^{2}}$, which in fact is constitutive for all field theories of massive particles. Here the four-momentum of the particle is denoted as $p^{\mu}=$ $(E, \mathbf{p})$, and the units are such that the speed of light $c=1$, and the particle mass is $m$. The contravariant four-momentum operator in space-time is denoted by $P^{\mu}=\left(P_{0}, \mathbf{P}\right)$, and its covariant form is given by the differential operator $P_{\mu}=\left(P_{0},-\mathbf{P}\right)=\mathrm{i} \partial_{\mu}=\mathrm{i}(\partial / \partial t, \partial / \partial \mathbf{x})$ (conventionally we use units of $\hbar=1$ ). The energy-momentum relation is usually written in manifestly covariant form as mass-shell condition, $p^{\mu} p_{\mu}=m^{2}$. Dirac's equation results from its linearization, which is achieved by introducing four new operators (usually represented by matrices), namely, the matrix three-vector $\boldsymbol{\alpha}$ and scalar $\beta$, yielding a Hamiltonian in the linear algebraic form

$$
\mathscr{l}=\beta m+\boldsymbol{\alpha} \cdot \mathbf{P} .
$$

As shown in any text book (see, e.g., $[9,10]$ ), in terms of matrices one requires at least four dimensions to represent the operators $\boldsymbol{\alpha}$ and $\beta$. Furthermore, to satisfy the mass-shell requirement they must algebraically obey the (indicated by the symbol $\{$,$\} ) anticommutator,$

$$
\{\alpha, \beta\}=0,
$$


and also have the property that $\beta^{2}=1$ and $(\boldsymbol{\alpha} \cdot \mathbf{p})^{2}=\mathbf{p}^{2}$. This is readily ensured, for example in the chiral representation, if we decompose the $4 \times 4$ matrix as follows, $\boldsymbol{\alpha}=\bar{\alpha} \sigma$, with $\bar{\alpha}^{2}=1$. Thus, $\boldsymbol{\alpha}$ can concisely be expressed in terms of the spin operator $\sigma$ and $\bar{\alpha}$. The $2 \times 2$ Pauli matrices are known to have the required property $(\boldsymbol{\sigma} \cdot \mathbf{p})^{2}=\mathbf{p}^{2}$. Similarly, we can write $\beta=\bar{\beta} \sigma_{0}$. Finally, we quote explicitly the two real matrices $\bar{\alpha}$ and $\bar{\beta}$ (and a related one called $\bar{\gamma}=\bar{\beta} \bar{\alpha}$ ), which are going to be used later and are defined as

$$
\bar{\alpha}=\left(\begin{array}{cc}
1 & 0 \\
0 & -1
\end{array}\right), \quad \bar{\beta}=\left(\begin{array}{ll}
0 & 1 \\
1 & 0
\end{array}\right), \quad \bar{\gamma}=\left(\begin{array}{cc}
0 & -1 \\
1 & 0
\end{array}\right) .
$$

The three Pauli matrices [11], to which we add here the $2 \times 2$ unit matrix denoted as $\sigma_{0}$, have their standard form but are quoted again because of their importance to what follows. They read

$$
\sigma_{\mathrm{x}}=\left(\begin{array}{cc}
0 & 1 \\
1 & 0
\end{array}\right), \quad \sigma_{\mathrm{y}}=\left(\begin{array}{cc}
0 & -i \\
i & 0
\end{array}\right), \quad \sigma_{\mathrm{z}}=\left(\begin{array}{cc}
1 & 0 \\
0 & -1
\end{array}\right)
$$

and build the three-vector $\sigma=\left(\sigma_{x}, \sigma_{y}, \sigma_{z}\right)$. We may also introduce the two four-vector forms of the Pauli matrices. They are defined according to Jehle [12] by the four vectors $\sigma_{ \pm}^{\mu}=\left(\sigma_{0}, \pm \boldsymbol{\sigma}\right)$ and obey $\sigma_{ \pm}^{\mu}=\sigma_{\mp \mu}$. Making use of the above matrices, we can obtain Dirac's equation in standard chiral form $[1,10]$ and the classical Hamiltonian representation.

Returning to the basic equation (2.1), the question may then be asked whether there are other than pure matrix representations of the operators $\boldsymbol{\alpha}$ and $\beta$. In a recent paper [1] it was shown that the answer is yes. As this subject is not common knowledge, we shall repeat here some of the key algebra, making use of the operator of complex conjugation $\mathbb{C}$, which transforms any complex number $z$ into its complex conjugate $z^{*}$. This appears naturally in the symmetry operations of time inversion and charge conjugation of Dirac's equation [10]. Therefore let us define an important operator, which is not of pure algebraic nature but involves this complex-conjugation operator $\mathbb{C}$. We call (in partial nomenclature analogy to $\beta$ ) this operator $\tau$ and define it appropriately as $\tau=\sigma_{\mathrm{y}} \mathbb{C}$, which differs from the definition in Marsch's paper [1] by an unimportant phase factor i. The importance of $\tau$ was first recognized by Case [6]. It is anti-Hermitian conjugate, $\tau^{\dagger}=-\tau$, and other than $\beta$ obeys the relation $\tau^{2}=-1$. Obviously, the operation of $\tau$ on the spin vector $\sigma$ leads to its inversion; that is, when using the commutation rules of the spin operators, we can show that the operation $\tau \sigma \tau^{-1}=-\sigma$ yields a spin flip. Also remember from (2.4) that $\sigma^{*}=\sigma^{\mathrm{T}}$, where the superscript $T$ indicates the transposed matrix. As $\tau$ flips the spin, we have $\tau \sigma_{ \pm}^{\mu}=\sigma_{\mp}^{\mu} \tau$, and $\tau \mathrm{i}+\mathrm{i} \tau=0$, because of the action of $\mathbb{C}$. Therefore, $\tau$ also anticommutes with the momentum four-vector operator $P_{\mu}$, and thus we have $\tau P_{0}+P_{0} \tau=0$ and $\tau \mathbf{P}+\mathbf{P} \tau=0$.

Making use of the previously mentioned properties of the spin flip operator $\tau=\sigma_{\mathrm{y}} \mathbb{C}$, we can now still use a two-component matrix representation given by the Pauli matrices (2.4). Therefore, by help of $\tau$ we can go a decisive mathematical step beyond pure matrix algebra and define the linear energy operator

$$
\mathscr{H}=\tau m+\boldsymbol{\sigma} \cdot \mathbf{P}
$$


in analogy to (2.1). The operators involved obey the anticommutation rule,

$$
\{\sigma, \tau\}=0,
$$

and also have the property that $(\boldsymbol{\sigma} \cdot \mathbf{p})^{2}=\mathbf{p}^{2}$, but $\tau^{2}=-1$. We stress again that for the spacetime operators $\{\mathbf{P}, \tau\}=0$ and $\left\{P_{0}, \tau\right\}=0$. When putting $P_{0}=\mathscr{H}$ in (2.5), squaring it and multiplying out, the previous key features of $\tau$ must be exploited. Thus we retain, when inserting the differential operators explicitly, the Klein-Gordon equation.

As a consequence of (2.5), and without recourse to the Dirac equation, we obtain directly a linear wave equation from (2.5), which is named after Weyl [13] without the mass term and after Majorana with the mass term. It involves only the Pauli matrix operators acting on a two-component spinor $\phi$ but introduces the complications that are caused by the operator $\tau$ and reads as follows:

$$
\mathrm{i}\left(\frac{\partial}{\partial t}+\boldsymbol{\sigma} \cdot \frac{\partial}{\partial \mathbf{x}}\right) \phi(\mathbf{x}, t)=m \tau \phi(\mathbf{x}, t)
$$

In (2.7) we have not indicated the $2 \times 2$-unit matrix explicitly. This equation is nothing but what is called nowadays the two-component Majorana equation $[4,5]$, although it was never written down this way by Majorana himself. As shown by case in [6], this equation can be also derived from Dirac's equation in chiral form, or more generally if one imposes on it the condition of Lorentz-covariant complex conjugation, a procedure which is clearly described in a recent tutorial paper of $\mathrm{Pal}$ [8]. However, the view we take here is that equally well we may consider (2.5) as basic, in the spirit of the citation [6] quoted in the introduction.

Obviously, there is a second version of the Majorana (2.7), which is obtained by the operation of $\tau$ on it. Namely, when we apply $\tau$ from the left side, we find that

$$
\mathrm{i}\left(\frac{\partial}{\partial t}-\boldsymbol{\sigma} \cdot \frac{\partial}{\partial \mathbf{x}}\right) \tau \phi(\mathbf{x}, t)=-m \tau(\tau \phi(\mathbf{x}, t))=m \phi(\mathbf{x}, t)
$$

Consequently, if $\phi$ solves (2.7) with the plus sign in front of $\sigma$, then $X=\tau \phi$ solves it with the minus sign, but with a minus sign also at the mass term. Effectively, this amounts to replacing $\boldsymbol{\sigma}$ by $-\sigma$ in (2.7), also implying $\tau$ is exchanged by $-\tau$.

Apparently, the two equations (2.7) and (2.8) are closely connected twins. It is interesting to note that the two together can provide a special but important four-component Dirac spinor in the form

$$
\psi_{ \pm}^{c}=\left(\begin{array}{c}
\phi \\
\pm \tau \phi
\end{array}\right)
$$

which solves the Dirac equation. Moreover, charge (or better chirality) conjugation $[9,10]$ is, in the chiral representation, given by the operator $C=\bar{\gamma} \tau$, which when operating on $\psi_{ \pm}^{\mathcal{C}}$ reproduces it. Since $\mathcal{C}^{2}=1$, this operator has only the eigenvalue \pm 1 . Therefore, the spinors $\psi_{ \pm}^{\mathcal{C}}$ constructed from the solution of the Majorana equation are the two eigenfunctions with eigenvalue \pm 1 of the chirality conjugation operator $\mathcal{C}$. Moreover, the chiral projection operators are given by $P_{ \pm}=1 / 2(1 \pm \bar{\alpha})$, and thus the left- and right-chiral spinor is $\psi_{\mathrm{R}, \mathrm{L}}=P_{ \pm} \psi$. 
As $\{\bar{\gamma}, \bar{\alpha}\}=0$, we obtain $\mathcal{C} P_{ \pm}=P_{\mp} \mathcal{C}$, and consequently, if one applies $\mathcal{C}$ separately to the rightor left-chiral fields, one obtains

$$
\mathcal{C}\left[\left(\psi^{c}\right)_{\mathrm{R}}\right]=\left(\psi^{\mathcal{C}}\right)_{\mathrm{L}^{\prime}} \quad \mathcal{C}\left[\left(\psi^{\mathcal{C}}\right)_{\mathrm{L}}\right]=\left(\psi^{\mathcal{C}}\right)_{\mathrm{R}}
$$

Thus this operator exchanges the chirality of the chiral projections of the complex-selfconjugate spinor $\psi^{c}$, and it transforms left- into right-handed states and vice versa, a virtue which is essentially associated with the spin-flip operator $\tau$ as key ingredient of $\mathcal{C}$.

In conclusion, in this special situation the operator $\mathcal{C}$ acts as chirality conjugation or reversal. While being a composite of the two related and by themselves irreducible Majorana equations, the reducible chiral Dirac equation has as an intrinsic symmetry of this chirality conjugation symmetry.

Consequently, in what follows we can interpret the spinor $\phi$ as representing a rightchiral field, and vice versa $\chi=\tau \phi$ as representing a left-chiral field, which is obtained from $\phi$ by application of the spin-flip operator. Apparently, it translates from one into the other irreducible representation of the Lorentz group.

Let us consider now the symmetries of the two-component complex Majorana equation, in particular the charge exchange $\mathcal{C}$, parity $D$, and time reversal $\tau$ operations. Here we expand the lucid discussion of case in [6]. Generally speaking the Majorana equation is invariant under the symmetry operation $\mathcal{O}$, if the two-component spinor

$$
\phi^{\mathcal{O}}=\mathcal{O} \phi
$$

also fulfils that equation. When applying the operation $\mathcal{O}$ from the left and its inverse $\mathcal{O}^{-1}$ from the right, whereby the unit operator is given by the decomposition $\mathcal{O O}^{-1}=1$, we obtain the result

$$
\left(\mathcal{O}\left(\mathrm{i} \frac{\partial}{\partial t}\right) \mathcal{O}^{-1}+\mathcal{O}\left(\sigma \cdot \mathrm{i} \frac{\partial}{\partial \mathbf{x}}\right) \mathcal{O}^{-1}-m \mathcal{O} \tau \mathcal{O}^{-1}\right) \mathcal{O} \phi(\mathbf{x}, t)=0
$$

We define as usually the time and space coordinate inversion operations $\mathbb{T}$ and $\mathbb{P}$ on a spinor $\phi$ by

$$
\begin{aligned}
& \mathbb{T} \phi(\mathbf{x}, t)=\phi(\mathbf{x},-t), \\
& \mathbb{P} \phi(\mathbf{x}, t)=\phi(-\mathbf{x}, t)
\end{aligned}
$$

and also recall the complex conjugation operation $\mathbb{C}$, which gives $\mathbb{C i} \mathbb{C}^{-1}=-i$ and yields

$$
\mathbb{C} \phi(\mathbf{x}, t)=\phi^{*}(\mathbf{x}, t) .
$$

Here the asterisk denotes again the complex conjugate number. With these preparations in mind, it is easy to see which operators provide the various symmetry operations, which are composed in the Table 1. To complete the operator algebra, it is important to note that the coordinate reversal operators $\mathbb{T}$ and $\mathbb{P}$ commute with $\tau$ and $\sigma$, respectively. 
Table 1: Symmetry operations.

\begin{tabular}{lccc}
\hline Operation & Time reversal & Parity & Chirality (charge) conjugation \\
\hline Operator & $\tau=\tau \mathbb{T}$ & $p=i \tau \mathbb{P}$ & $\mathcal{C}=\tau=\sigma_{\mathrm{y}} \mathbb{C}$ \\
\hline
\end{tabular}

Let us first consider in (2.12) the time reversal, $\mathcal{O}=\tau=\tau \mathbb{T}$. Apparently, it does not affect the mass term with $\tau$ and also leaves the first term as well as the kinetic term i $\sigma$ unchanged. Therefore, $\phi^{\tau}=\sigma_{\mathrm{y}} \phi^{*}(\mathbf{x},-t)$ solves the Majorana equation (2.7) as well. Conversely, the parity operation $\mathcal{O}=D=\mathrm{i} \tau \mathbb{P}$ anticommutes with the mass term and inverts the sign of the first term in (2.12), and it also does not leave the momentum term invariant, since as $\mathbf{x}$ changes its sign, so does the spatial derivative. Consequently, $\phi^{D}=\mathrm{i} \sigma_{\mathrm{y}} \phi^{*}(-\mathbf{x}, t)$ does solve the Majorana equation (2.7). Finally, we consider the chirality conjugation, $\mathcal{O}=\mathcal{C}=\tau$, which only changes the sign of the first term. Therefore, $\phi^{C}=\sigma_{\mathrm{y}} \phi^{*}(\mathbf{x}, t)$ does not solve the Majorana equation (2.7) but solves its conjugate version (2.8). In conclusion, of the symmetry operations given in Table 1 time reversal and parity are obeyed, but chirality inversion is maximally broken.

Finally, as the second major topic of our paper we shall reformulate the twocomponent Majorana equation in its real form by decomposing the spinor $\phi$ into its real and imaginary part: $\phi=\phi_{R}+\mathrm{i} \phi_{I}$. Making use of the three real $2 \times 2$ matrices defined in (2.3) we can write:

$$
\left(\bar{\gamma} \frac{\partial}{\partial t}-\bar{\alpha} \frac{\partial}{\partial x}+\bar{\beta} \frac{\partial}{\partial z}\right) \phi_{R}+\frac{\partial}{\partial y} \phi_{I}=-m \phi_{R}
$$

and similarly we obtain

$$
\left(\bar{\gamma} \frac{\partial}{\partial t}-\bar{\alpha} \frac{\partial}{\partial x}+\bar{\beta} \frac{\partial}{\partial z}\right) \phi_{I}-\frac{\partial}{\partial y} \phi_{R}=+m \phi_{I}
$$

both of which can be combined, by introducing $\phi_{ \pm}=\phi_{R} \pm \phi_{I}$, in a single equation:

$$
\left(\bar{\gamma} \frac{\partial}{\partial t}-\bar{\alpha} \frac{\partial}{\partial x}+\bar{\beta} \frac{\partial}{\partial z}\right) \phi_{ \pm}=\left( \pm \frac{\partial}{\partial y}-m\right) \phi_{\mp}
$$

Note that $\bar{\gamma}^{2}=-1, \bar{\alpha}^{2}=1$, and $\bar{\beta}^{2}=1$. Furthermore, these matrices all anticommute, and thus by squaring (2.17) one immediately retains the scalar Klein-Gordon equation reading

$$
\left(\frac{\partial^{2}}{\partial t^{2}}-\frac{\partial^{2}}{\partial \mathbf{x}^{2}}+m^{2}\right) \phi_{ \pm}=0
$$

which in fact was our starting point, when deriving a relativistic wave equation.

From the two real two-component Majorana equations (2.17), when both are combined into a single one, the standard Dirac equation in the real Majorana representation follows immediately. Namely, we may arrange the two spinors $\phi_{ \pm}$into a single four-component real 
Dirac spinor and write $\psi^{\dagger}=\left(\phi_{+}^{\dagger}, \phi_{-}^{\dagger}\right)$. Then the coupled system of (2.17) transforms into a $4 \times 4$ real matrix differential equation which reads

$$
\left(\begin{array}{ll}
\bar{\gamma} & 0 \\
0 & \bar{\gamma}
\end{array}\right) \frac{\partial \psi}{\partial t}-\left(\begin{array}{cc}
\bar{\alpha} & 0 \\
0 & \bar{\alpha}
\end{array}\right) \frac{\partial \psi}{\partial x}+\left(\begin{array}{cc}
0 & -1 \\
1 & 0
\end{array}\right) \frac{\partial \psi}{\partial y}+\left(\begin{array}{cc}
\bar{\beta} & 0 \\
0 & \bar{\beta}
\end{array}\right) \frac{\partial \psi}{\partial z}=-m\left(\begin{array}{ll}
0 & 1 \\
1 & 0
\end{array}\right) \psi .
$$

Consequently, we may now introduce the subsequent real $4 \times 4$ Majorana matrices in their natural (as deduced from the Pauli matrices) representation:

$$
\bar{\gamma}^{\mu}=\left(\left(\begin{array}{cc}
0 & \bar{\gamma} \\
\bar{\gamma} & 0
\end{array}\right),\left(\begin{array}{cc}
0 & -\bar{\alpha} \\
-\bar{\alpha} & 0
\end{array}\right),\left(\begin{array}{cc}
1 & 0 \\
0 & -1
\end{array}\right),\left(\begin{array}{cc}
0 & \bar{\beta} \\
\bar{\beta} & 0
\end{array}\right)\right)
$$

They mutually anticommute and obey $\bar{\gamma}^{\mu} \bar{\gamma}^{\nu}+\bar{\gamma}^{\nu} \bar{\gamma}^{\mu}=-2 g^{\mu \nu}$. Thus the real Dirac equation in Majorana representation reads

$$
\bar{\gamma}^{\mu} \partial_{\mu} \psi+m \psi=0
$$

which can, with the help of the purely imaginary Dirac matrices $\gamma^{\mu}=\mathrm{i} \bar{\gamma}^{\mu}$, easily be brought into the standard form of the Dirac equation:

$$
\mathrm{i} \gamma^{\mu} \partial_{\mu} \psi=m \psi
$$

Therefore, the four-component Dirac equation (2.21) is, in its real Majorana representation, a direct consequence of the basic two-component complex Majorana equation (2.7), which was derived here without invoking the Dirac equation in the first place. Subsequently, we shall also derive the four-component real eigenspinors of (2.21).

\section{Eigenfunctions of the Two-Component Majorana Equation with Mass Term}

Returning to the basic equation (2.7), we here derive its eigenfunctions. In order to solve it, we make the usual plane-wave ansatz, $\phi(\mathbf{x}, t)=u(\mathbf{p}, E) \exp (-\mathrm{i} E t+\mathrm{ip} \cdot \mathbf{x})+v(\mathbf{p}, E) \exp (\mathrm{i} E t-\mathrm{ip} \cdot \mathbf{x})$. But note that we require the plane wave and its complex conjugate as well, because of the operator $\tau$ in (2.7). The two-component spinors $u$ and $v$ cannot be assumed to be complex conjugated; that is, the wave function cannot be expected to be real. The resulting linked eigenspinor equations are

$$
\begin{gathered}
(E-\boldsymbol{\sigma} \cdot \mathbf{p}) u(\mathbf{p}, E)=m \tau v(\mathbf{p}, E) \\
(E-\boldsymbol{\sigma} \cdot \mathbf{p}) v(\mathbf{p}, E)=-m \tau u(\mathbf{p}, E)
\end{gathered}
$$


By insertion of the first into the second equation, or vice versa, the relativistic dispersion relation is obtained:

$$
\left(\tau(E-\boldsymbol{\sigma} \cdot \mathbf{p}) \tau(E-\boldsymbol{\sigma} \cdot \mathbf{p})+m^{2}\right) u(\mathbf{p}, E)=0
$$

which yields the two eigenvalues

$$
E_{1,2}(\mathbf{p})= \pm \sqrt{m^{2}+\mathbf{p}^{2}}
$$

which are obtained from the requirement that for nontrivial solutions of the spinors $u$ and $v$ to exist the determinant associated with the eigenvalue problem (3.3) must vanish. The negative root in (3.4) cannot be neglected, since as usually it is related to antiparticles. We can solve (3.2) for $v$ and insert it back into the previous ansatz for $\phi(\mathbf{x}, t)$ to obtain finally the solutions of (2.7) and (2.8) in the concise forms:

$$
\begin{gathered}
\phi(\mathbf{x}, t)=\left(1-\frac{E+\boldsymbol{\sigma} \cdot \mathbf{p}}{m} \tau\right) \exp (-\mathrm{i} E t+\mathrm{i} \mathbf{p} \cdot \mathbf{x}) u, \\
x(\mathbf{x}, t)=\tau \phi(\mathbf{x}, t)=\left(\tau+\frac{E-\boldsymbol{\sigma} \cdot \mathbf{p}}{m}\right) \exp (-\mathrm{i} E t+\mathrm{i} \mathbf{p} \cdot \mathbf{x}) u .
\end{gathered}
$$

To validate these solutions by direct differentiation, careful attention must be paid to the anticommutation rules between $\tau$ and i, respectively, $\sigma$. We may also solve (3.2) for $u$ instead, and then insert it back into the previous ansatz for $\phi(x, t)$.

We are free to choose for the eigenspinor $u$ the two standard spin-up and -down eigenfunctions: $u_{1}^{\dagger}=(1,0)$ and $u_{2}^{\dagger}=(0,1)$, and similarly for $v$, but there is a better and more adequate choice if $p$ is nonzero, as discussed in the following. Similar solutions like that of (3.5) and (3.6) are obtained for the negative energy root in (3.1), yielding the antiparticle wavefunctions. Superposition of all the Fourier modes and their summation over the momentum variable $\mathbf{p}$ leads finally to the general Majorana fields, whose quantization then follows from the canonical rules [2-5] and is given in the following. Above we obtained the formal solutions of the Majorana equation, which we reiterate here by introducing the Majorana operator $\mathcal{M}$ as follows:

$$
\mathcal{M}=\mathrm{i}\left(\frac{\partial}{\partial t}+\boldsymbol{\sigma} \cdot \frac{\partial}{\partial \mathbf{x}}\right)-m \tau
$$

The solution $\phi$ obeys $\mathcal{M} \phi=0$. Note, however, that the four-momentum operator $P_{\mu}=\mathrm{i} \partial_{\mu}=$ $\mathrm{i}(\partial / \partial t, \partial / \partial \mathbf{x})$ does not commute with $\mathcal{M}$, and neither does the spin-flip operator $\tau$. Therefore, the $\phi$ and $\chi$ of (3.5) to (3.6) are not eigenfunctions of any of these operators. However, the helicity operator, $\boldsymbol{\sigma} \cdot \mathbf{P}$, does commute with $\mathcal{M}$.

Consequently, to chose the eigenfunctions of the helicity operator in $\phi$ for the free functions $u$ or $v$ is most convenient. The eigenvalue equation of the helicity operator in Fourier space reads

$$
(\sigma \cdot \widehat{\mathbf{p}}) u_{ \pm}(\widehat{\mathbf{p}})= \pm u_{ \pm}(\widehat{\mathbf{p}})
$$


The two eigenvectors depend on the momentum unit vector $\widehat{\mathbf{p}}=\mathbf{p} / p$ and are given by

$$
u_{+}(\widehat{\mathbf{p}})=\left(\begin{array}{c}
\cos \frac{\theta}{2} \mathrm{e}^{-(\mathrm{i} / 2) \phi} \\
\sin \frac{\theta}{2} \mathrm{e}^{(\mathrm{i} / 2) \phi}
\end{array}\right), \quad u_{-}(\widehat{\mathbf{p}})=\left(\begin{array}{c}
-\sin \frac{\theta}{2} \mathrm{e}^{-(\mathrm{i} / 2) \phi} \\
\cos \frac{\theta}{2} \mathrm{e}^{(\mathrm{i} / 2) \phi}
\end{array}\right),
$$

in which the half angles of $\theta$ and $\phi$ appear. The eigenvectors for the same $\widehat{\mathbf{p}}$ are orthogonal to each other and normalized to a modulus of unity, and they obey the relation $u_{ \pm}(-\widehat{\mathbf{p}})=$ $u_{\mp}(\widehat{\mathbf{p}})$. This is a consequence of the eigenvalue equation (3.8), which implies that $u_{ \pm}(\widehat{\mathbf{p}})$ is an eigenvector of the helicity operator, corresponding to a right-handed, respectively, left-handed, screw with respect to the momentum direction. According to (3.9) we have $u_{ \pm}^{\dagger}(\widehat{\mathbf{p}}) u_{ \pm}(\widehat{\mathbf{p}})=1$ and $u_{\mp}^{\dagger}(\widehat{\mathbf{p}}) u_{ \pm}(\widehat{\mathbf{p}})=0$. The dagger denotes as usually the transposed (denoted by the superscript $T$ ) and complex conjugated vector, respectively, matrix. The scalar product between two vectors (spinors) $v$ and $w$ is just the sum over the products of their two components; that is, $v w$ simply stands for $v_{1} w_{1}+v_{2} w_{2}$.

We emphasize that the spin-flip operator $\tau=\sigma_{y} \mathbb{C}$, when operating on the previous eigenspinors, leads to

$$
\tau u_{ \pm}(\widehat{\mathbf{p}})= \pm \mathrm{i} u_{\mp}(\widehat{\mathbf{p}})
$$

That is, it connects the eigenfunctions of opposite helicity and turns out to be a quite useful relation in the subsequent considerations. By its application, we can write the two possible associated eigenfunctions after (3.5) as

$$
\phi_{ \pm}(\mathbf{x}, t)=\exp (-\mathrm{i} E t+\mathrm{i} \mathbf{p} \cdot \mathbf{x}) u_{ \pm}(\widehat{\mathbf{p}}) \mp \frac{\mathrm{i}}{m}(E+\boldsymbol{\sigma} \cdot \mathbf{p}) \exp (\mathrm{i} E t-\mathrm{i} \mathbf{p} \cdot \mathbf{x}) u_{\mp}(\widehat{\mathbf{p}})
$$

where the advantage of the helicity eigenfunctions becomes obvious. Namely, by use of (3.8) we obtain the (now normalized to unity) eigenfunction

$$
\phi_{ \pm}(\mathbf{x}, t)=\frac{1}{\sqrt{2 E}}\left(\sqrt{E \pm p} \exp (-\mathrm{i} E t+\mathrm{i} \mathbf{p} \cdot \mathbf{x}) u_{ \pm}(\widehat{\mathbf{p}}) \mp \mathrm{i} \sqrt{E \mp p} \exp (\mathrm{i} E t-\mathrm{i} \mathbf{p} \cdot \mathbf{x}) u_{\mp}(\widehat{\mathbf{p}})\right)
$$

which is a mixed state involving both helicities in a symmetric fashion. Note that for vanishing mass, $m=0$ corresponding to the Weyl equation, only the positive helicity remains, and thus the wavefunction becomes purely right-handed. Its left-handed version is then obtained by applying the spin-flip operator $\tau$ on (3.12) like in (3.6). Operation of $\mathcal{M}$ on this $\phi_{ \pm}$validates that it solves the Majorana equation, that is, $\mathcal{M} \phi_{ \pm}=0$. A simple form of the eigenspinor is obtained for a particle at rest, that is, $p=0$, which yields

$$
\phi_{ \pm}(t)=\frac{1}{\sqrt{2}}\left(\exp (-\mathrm{i} m t) u_{ \pm} \mp \mathrm{i} \exp (+\mathrm{i} m t) u_{\mp}\right) .
$$

The helicity eigenvectors in this case can be chosen to be the standard ones obtained for the angles $\phi=0$ and $\theta=0$ according to (3.9). 
Concerning the adequate choice of the eigenspinor $u_{1,2}$ in (3.5) for the two possible eigenvalues $E_{1,2}(p)= \pm \sqrt{m^{2}+p^{2}}$, we recall that the helicity operator does commute with $\mathcal{M}$, and generally we should consider a mixture or superposition of both helicities. But this is indeed already implied in the solution (3.12). Therefore, as we can only have two linearly independent eigenvectors to define the basis, we assume that $u$ can be decomposed such that these two eigenvectors are defined by

$$
u_{1,2}(\mathbf{p})=a_{1,2}(\mathbf{p}) u_{ \pm}(\widehat{\mathbf{p}})
$$

with some complex amplitude $a_{1,2}(\mathbf{p})$ of module unity, $\left|a_{1,2}\right|^{2}=1$, to ensure normalization. The natural but arbitrary association with the sign of the energy (3.4) is to take the positive sign for positive (right-handed) helicity and the negative sign for negative (left-handed) helicity. Thus we may conventionally refer to particles (index 1) and antiparticles (index 2). Upon insertion of this ansatz in (3.5) we obtain a yet more general expression for the two related eigenfunctions as follows:

$$
\begin{aligned}
& \phi_{1}(\mathbf{x}, t)=\sqrt{\frac{E_{1}+p}{2 E_{1}}} \exp \left(-\mathrm{i} E_{1} t+\mathrm{i} \mathbf{p} \cdot \mathbf{x}\right) a_{1} u_{+}-\mathrm{i} \sqrt{\frac{E_{1}-p}{2 E_{1}}} \exp \left(\mathrm{i} E_{1} t-\mathrm{i} \mathbf{p} \cdot \mathbf{x}\right) a_{1}^{*} u_{-}, \\
& \phi_{2}(\mathbf{x}, t)=\sqrt{\frac{E_{2}-p}{2 E_{2}}} \exp \left(-\mathrm{i} E_{2} t+\mathrm{ip} \cdot \mathbf{x}\right) a_{2} u_{-}+\mathrm{i} \sqrt{\frac{E_{2}+p}{2 E_{2}}} \exp \left(\mathrm{i} E_{2} t-\mathrm{ip} \cdot \mathbf{x}\right) a_{2}^{*} u_{+},
\end{aligned}
$$

where the obvious momentum arguments in $u$ and $a$ have been suppressed for the sake of lucidity. Apparently, the previous wavefunction (3.12) is retained for $a_{1,2}(\mathbf{p})=1$, and $E_{1,2}=E$. It turns out to be convenient to introduce the two real quantities

$$
\varepsilon_{ \pm}(p)=\sqrt{\frac{E(p) \pm p}{2 E(p)}},
$$

the squares of which add up to unity, $\varepsilon_{+}^{2}+\varepsilon_{-}^{2}=1$. A useful property of the epsilons is the obvious relation $(E \pm p) \varepsilon_{\mp}=m \varepsilon_{ \pm}$. From now on $E(p)=\sqrt{m^{2}+p^{2}}$ is used for the positive root obtained from (3.4), and the argument $p$ in the epsilons will subsequently be omitted for simplicity. Then we can write the two eigenfunctions (with a new name) concisely as

$$
\begin{aligned}
& \tilde{\phi}_{1}(\mathbf{x}, t)=\varepsilon_{+} \exp (-\mathrm{i} E t+\mathrm{i} \mathbf{p} \cdot \mathbf{x}) a_{1} u_{+}-\mathrm{i} \varepsilon_{-} \exp (+\mathrm{i} E t-\mathrm{i} \mathbf{p} \cdot \mathbf{x}) a_{1}^{*} u_{-} \\
& \tilde{\phi}_{2}(\mathbf{x}, t)=\varepsilon_{+} \exp (+\mathrm{i} E t+\mathrm{i} \mathbf{p} \cdot \mathbf{x}) a_{2} u_{-}+\mathrm{i} \varepsilon_{-} \exp (-\mathrm{i} E t-\mathrm{i} \mathbf{p} \cdot \mathbf{x}) a_{2}^{*} u_{+}
\end{aligned}
$$

To make the antiparticle wavefunction having the same standard plane wave phase like that of the particle, we invert its momentum and note again that the polarization vectors change according to $u_{ \pm}(-\widehat{\mathbf{p}})=u_{\mp}(\widehat{\mathbf{p}})$. This inversion is permitted as we will later sum over all momenta, and thus $\mathbf{p}$ is just a mute index. Consequently, we take $\phi_{\mathrm{P}}(\mathbf{p})=\tilde{\phi}_{1}(\mathbf{p})$ for the particle, but $\phi_{\mathrm{A}}(\mathbf{p})=\widetilde{\phi}_{2}(-\mathbf{p})$ for the antiparticle, and we redefine the amplitudes as 
$a(\mathbf{p})=a_{1}(\mathbf{p})$ for the particle, but $b(\mathbf{p})=\mathrm{i} a_{2}^{*}(-\mathbf{p})$, respectively, $b^{*}(\mathbf{p})=-\mathrm{i} a_{2}(-\mathbf{p})$ for the antiparticle. The new wavefunctions finally read as follows:

$$
\begin{aligned}
& \phi_{\mathrm{P}}(\mathbf{x}, t)=\varepsilon_{+} \exp (-\mathrm{i} E t+\mathrm{i} \mathbf{p} \cdot \mathbf{x}) a u_{+}-\mathrm{i} \varepsilon_{-} \exp (+\mathrm{i} E t-\mathrm{i} \mathbf{p} \cdot \mathbf{x}) a^{*} u_{-} \\
& \phi_{\mathrm{A}}(\mathbf{x}, t)=\varepsilon_{-} \exp (-\mathrm{i} E t+\mathrm{i} \mathbf{p} \cdot \mathbf{x}) b u_{-}+\mathrm{i} \varepsilon_{+} \exp (+\mathrm{i} E t-\mathrm{i} \mathbf{p} \cdot \mathbf{x}) b^{*} u_{+} .
\end{aligned}
$$

Both eigenfunctions are normalized to unity, yielding the relation $\phi_{\mathrm{P}, \mathrm{A}}^{\dagger} \phi_{\mathrm{P}, \mathrm{A}}=1$, which is obtained by using $\varepsilon_{+}^{2}+\varepsilon_{-}^{2}=1$, exploiting the normalization and orthogonality of the helicity eigenvectors, and noting that $a^{*} a=1$ and $b^{*} b=1$. They form a complete eigenvector basis of the Majorana operator $\mathcal{M}$ and obey $\mathcal{M} \phi_{\mathrm{P}, \mathrm{A}}=0$. However, we emphasize again that they are neither eigenfunctions of the four-momentum operator $P^{\mu}=\left(P_{0}, \mathbf{P}\right)$ nor of the helicity operator or the spin-reversal operator $\tau$.

By adding up the two contributions we retain the most general solution of the Majorana equation (2.7) as the two-component spinor in the form

$$
\phi(\mathbf{x}, t)=\phi_{\mathrm{P}}(\mathbf{x}, t)+\phi_{\mathrm{A}}(\mathbf{x}, t) .
$$

This superposition of the particle and antiparticle eigenspinors yields the full Majorana wavefunction in the form

$$
\phi(\mathbf{x}, t)=\exp (-\mathrm{i} E t+\mathrm{i} \mathbf{p} \cdot \mathbf{x})\left(\varepsilon_{+} u_{+} a+\varepsilon_{-} u_{-} b\right)-\mathrm{i} \exp (+\mathrm{i} E t-\mathrm{i} \mathbf{p} \cdot \mathbf{x})\left(\varepsilon_{-} u_{-} a^{*}-\varepsilon_{+} u_{+} b^{*}\right) .
$$

\section{The Majorana Quantum Field}

At this point the transition from (3.20) to a quantum field is quite natural and obvious. We just have to replace the amplitudes in (3.20) by the canonical anticommuting fermion operators obeying

$$
\begin{aligned}
& \left\{a(\mathbf{p}), a^{\dagger}\left(\mathbf{p}^{\prime}\right)\right\}=\delta_{\mathbf{p}, \mathbf{p}^{\prime}} \\
& \left\{b(\mathbf{p}), b^{\dagger}\left(\mathbf{p}^{\prime}\right)\right\}=\delta_{\mathbf{p}, \mathbf{p}^{\prime}}
\end{aligned}
$$

Of course, all possible anticommutators between two creation and two annihilation operators are zero. Mutually, between the $a$ s and $b$ s the anticommutators vanish, as they should since these two degrees of freedom are independent. The operator $a^{\dagger}(\mathbf{p})$ creates, and vice versa $a(\mathbf{p})$ annihilates the plane-wave state of a particle of positive helicity, with momentum $\mathbf{p}$ and energy $E=\sqrt{m^{2}+p^{2}}$. The $b$-operators do the same, yet for the related antiparticle of the opposite negative helicity.

We finally get, by keeping explicitly all momentum arguments, the following field operator for any given momentum $\mathbf{p}$ in the concise form

$$
\Phi_{\mathbf{p}}(\mathbf{x}, t)=\exp (-\mathrm{i} E(p) t+\mathrm{ip} \cdot \mathbf{x}) \underline{c}(\mathbf{p})-\mathrm{i} \exp (+\mathrm{i} E(p) t-\mathrm{ip} \cdot \mathbf{x}) \underline{d}^{\dagger}(\mathbf{p}),
$$


which assumes the standard form (similar to what is known from the Dirac equation). The quantum field operators corresponding to $\phi$ are denoted by a capital $\Phi$. We can now sum up overall momenta to obtain the full Majorana quantum field:

$$
\Phi_{\mathcal{M}}(\mathbf{x}, t)=\sum_{\mathbf{p}} \Phi_{\mathbf{p}}(\mathbf{x}, t)
$$

We introduced as abbreviations two operators, which correspond to the creation, respectively, annihilation, polarization-vector operators (indicated by an underscore) for a given mixed helicity state as follows:

$$
\underline{c}(\mathbf{p})=\varepsilon_{+}(p) u_{+}(\widehat{\mathbf{p}}) a(\mathbf{p})+\varepsilon_{-}(p) u_{-}(\widehat{\mathbf{p}}) b(\mathbf{p}),
$$

and similarly

$$
\underline{d}^{\dagger}(\mathbf{p})=\varepsilon_{-}(p) u_{-}(\widehat{\mathbf{p}}) a^{\dagger}(\mathbf{p})-\varepsilon_{+}(p) u_{+}(\widehat{\mathbf{p}}) b^{\dagger}(\mathbf{p})
$$

They obey the standard anticommutation rule, whereby the inner product between the original helicity eigenvector is used, as well as the relation $\varepsilon_{+}^{2}+\varepsilon_{-}^{2}=1$. Thus we obtain, by implying the standard scalar product between the complex two-component spinors involved, the result

$$
\left\{\underline{c}(\mathbf{p}), \underline{c}^{\dagger}\left(\mathbf{p}^{\prime}\right)\right\}=\delta_{\mathbf{p}, \mathbf{p}^{\prime}}
$$

and correspondingly

$$
\left\{\underline{d}(\mathbf{p}), \underline{d}^{\dagger}\left(\mathbf{p}^{\prime}\right)\right\}=\delta_{\mathbf{p}, \mathbf{p}^{\prime}}
$$

Again all possible mixed anticommutators between the $\underline{c}$ and $\underline{d}$ operators vanish. For the two particle number operators we obtain from (4.4) and (4.5) the linear combinations

$$
\begin{aligned}
& \underline{c}^{\dagger} \underline{c}=\varepsilon_{+}^{2} a^{\dagger} a+\varepsilon_{-}^{2} b^{\dagger} b, \\
& \underline{d}^{\dagger} \underline{d}=\varepsilon_{-}^{2} a^{\dagger} a+\varepsilon_{+}^{2} b^{\dagger} b .
\end{aligned}
$$

This combined coordinate transformation, involving the original eigenspinors of the helicity operator and their corresponding state operators (classically amplitudes), has a physically important interpretation. As stressed before, the helicity operator does commute with $\mathcal{M}$, but one requires a mixed helicity state to represent its eigenfunctions. This mixing depends on the ratio of the momentum, $p$, to the total energy, $E(p)$, of a particle. Only if $m=0$, we have pure left- or right-handed states, otherwise the massive states are mixed. That $\mathcal{M} \Phi_{\mathbf{p}}=0$ follows readily from the notion that

$$
(E-\boldsymbol{\sigma} \cdot \mathbf{p}) \underline{\underline{C}}(\mathbf{p})=m\left(\varepsilon_{-}(p) u_{+}(\widehat{\mathbf{p}}) a(\mathbf{p})+\varepsilon_{+}(p) u_{-}(\widehat{\mathbf{p}}) b(\mathbf{p})\right)=+m \mathbf{i} \tau \underline{d^{\dagger}}(\mathbf{p}),
$$


respectively, that

$$
(E-\boldsymbol{\sigma} \cdot \mathbf{p}) \underline{d}^{\dagger}(\mathbf{p})=m\left(\varepsilon_{+}(p) u_{-}(\widehat{\mathbf{p}}) a^{\dagger}(\mathbf{p})-\varepsilon_{-}(p) u_{+}(\widehat{\mathbf{p}}) b^{\dagger}(\mathbf{p})\right)=-m \mathbf{i} \tau \underline{c}(\mathbf{p}),
$$

where the auxiliary relation $(E \pm p) \varepsilon_{\mp}=m \varepsilon_{ \pm}$has been used. We should mention here that according to its definition the operation of $\tau$ on a creation or annihilation operator is defined such that $\tau a=a^{\dagger} \tau$, or similarly $\tau a^{\dagger}=a \tau$.

As an interlude, we may now consider the unmixed massless case $m=0$, which gives us the Weyl field. Then $\varepsilon_{+}=1$ and $\varepsilon_{-}=0$. The resulting quantum field operator is given by

$$
\Phi_{\mathcal{N R}}(\mathbf{x}, t)=\sum_{\mathbf{p}} u_{+}(\widehat{\mathbf{p}})\left(\exp (-\mathrm{i} p t+\mathrm{i} \mathbf{p} \cdot \mathbf{x}) a(\mathbf{p})+\mathrm{i} \exp (+\mathrm{i} p t-\mathrm{i} \mathbf{p} \cdot \mathbf{x}) b^{\dagger}(\mathbf{p})\right)
$$

Apparently, this field involves only the polarization vector for positive helicity. Therefore, in this case the right-chiral Weyl field operator annihilates right-handed particles and creates left-handed antiparticles. Operating with the spin flip operator $\tau$, according to $\tau a \tau^{-1}=a^{\dagger}$ and (3.10), on this field yields the left-chiral Weyl field, which reads

$$
\Phi_{\mathcal{L L}}(\mathbf{x}, t)=\sum_{\mathbf{p}} u_{-}(\widehat{\mathbf{p}})\left(\exp (-\mathrm{i} p t+\mathrm{i} \mathbf{p} \cdot \mathbf{x}) b(\mathbf{p})+\mathrm{i} \exp (+\mathrm{i} p t-\mathrm{i} \mathbf{p} \cdot \mathbf{x}) a^{\dagger}(\mathbf{p})\right)
$$

Consequently, in the case of the left-chiral Weyl field the operator creates right-handed particles and annihilates left-handed antiparticles.

Similarly, we obtain for the massive right-chiral Majorana quantum field:

$$
\Phi_{\mathscr{M R}}(\mathbf{x}, t)=\sum_{\mathbf{p}}\left(\exp (-\mathrm{i} E(p) t+\mathrm{i} \mathbf{p} \cdot \mathbf{x}) \underline{c}(\mathbf{p})-\mathrm{i} \exp (+\mathrm{i} E(p) t-\mathrm{i} \mathbf{p} \cdot \mathbf{x}) \underline{d}^{\dagger}(\mathbf{p})\right)
$$

and by application of $\tau$ on it the left-chiral Majorana quantum field:

$$
\Phi_{\mathcal{M L}}(\mathbf{x}, t)=\sum_{\mathbf{p}}\left(\exp (-\mathrm{i} E(p) t+\mathrm{i} \mathbf{p} \cdot \mathbf{x})\left(\mathrm{i} \tau \underline{d}^{\dagger}(\mathbf{p})\right)-\mathrm{i} \exp (+\mathrm{i} E(p) t-\mathrm{i} \mathbf{p} \cdot \mathbf{x})(\mathrm{i} \tau \underline{c}(\mathbf{p}))\right) .
$$

The corresponding operators can be read off as in (4.9) and (4.10).

Ultimately, we are interested in the expectation value of a given Hermitian operator $\mathcal{O}$ for the quantum field $\Phi$, for example, the Majorana fields (4.13) and (4.14) or the Weyl fields of (4.11) and (4.12), which are all not Hermitian fields. For the massless Weyl fields, the related plane waves are also eigenfunctions of the four-momentum operator $P^{\mu}$. In contrast, for the massive Majorana field this is not true, and therefore only the expectation value of $P^{\mu}$ can be evaluated. The same comment applies to the kinetic helicity operator, which yet does commute with $\mathcal{M}$. This interesting feature was discussed at length and clarified by Mannheim [7], who concluded that for the massive Majorana field theory "second quantization is necessary a priori in order to produce a sensible particle interpretation." Therefore, we can 
only calculate average expectation values, which are defined by volume integrals of the real part of the binary form

$$
O=\langle\mathcal{O}\rangle=\frac{1}{2} \int d^{3} x\left(\Phi^{\dagger} \mathcal{O} \Phi+(\mathcal{O} \Phi)^{\dagger} \Phi\right)
$$

This formula shall be now applied to calculate the expectation value of the energymomentum four-vector, the helicity and number operator of the right-chiral Majorana quantum field $\Phi_{\mathcal{M R}}(\mathbf{x}, t)$ of (4.13). Upon its insertion into (4.15), and after some lengthy but standard calculations (similar to what is usually done with the Dirac equation, e.g., see the textbooks [10] or [9]) we obtain the concise result

$$
P_{\mathcal{M R}}^{\mu}=\left\langle\left(P_{0}, \mathbf{P}\right)\right\rangle_{\mathcal{M R}}=\sum_{\mathbf{p}}\left(\sqrt{m^{2}+p^{2}}, \mathbf{p}\right)\left(a^{\dagger}(\mathbf{p}) a(\mathbf{p})+b^{\dagger}(\mathbf{p}) b(\mathbf{p})\right)
$$

where conventionally an infinite constant, $-\sum_{\mathbf{p}} E(p)$, has been discarded as irrelevant zeropoint energy. Let us consider the helicity density at a given $\mathbf{p}$ of the Majorana operator (4.2). We find

$$
\Phi_{\mathbf{p}}^{\dagger}(\boldsymbol{\sigma} \cdot \widehat{\mathbf{p}}) \Phi_{\mathbf{p}}=\underline{c}^{\dagger}(\mathbf{p})(\boldsymbol{\sigma} \cdot \widehat{\mathbf{p}}) \underline{c}(\mathbf{p})+\underline{d}(\mathbf{p})(\boldsymbol{\sigma} \cdot \widehat{\mathbf{p}}) \underline{d}^{\dagger}(\mathbf{p})=a^{\dagger} a-b^{\dagger} b-\varepsilon_{-}^{2}+\varepsilon_{+}^{2} .
$$

and thus by using these relations and by summing up all contributions of (4.17), we obtain the total helicity operator $H_{\mathcal{M R}}$, which is (within an unimportant constant) given by

$$
H_{\mathcal{N R}}=\langle\boldsymbol{\sigma} \cdot \widehat{\mathbf{P}}\rangle_{\mathcal{N R}}=\sum_{\mathbf{p}}\left(a^{\dagger}(\mathbf{p}) a(\mathbf{p})-b^{\dagger}(\mathbf{p}) b(\mathbf{p})\right) .
$$

It involves the difference of the number operators of the particles and antiparticles, which therefore are expected to contribute oppositely to the net helicity.

Interestingly, we can go through the same procedure with the left-chiral field (4.14) and end up with exactly the same expectation values as calculated previously. One gets a similar result for the Weyl quantum field, by putting either $m=0$ in the Majorana field or by using directly (4.11) and (4.12). In other words it suffices to consider mathematically the original and constitutive equation (2.5). Chirality conjugation, that is, the replacement of the spin $\boldsymbol{\sigma}$ by its negative inverted vector $-\boldsymbol{\sigma}$, does not yield new physical information concerning the above expectation values (4.16) and (4.18).

\section{Four-Component Eigenspinors of the Majorana Equation}

Following the derivation of the real Majorana equation (2.21), its eigenfunctions can, by construction according to (2.19), easily be derived. Starting point is the complex twocomponent original wave function (3.20), in which the wave amplitudes $a$ and $b$ correspond after the previous section to particles, respectively, antiparticles, and thus transform into the 
related annihilation operators for the quantum fields. The four-component eigenfunction is therefore given by the spinor

$$
\psi=\left(\begin{array}{c}
\phi_{\mathrm{R}}+\phi_{\mathrm{I}} \\
\phi_{\mathrm{R}}-\phi_{\mathrm{I}}
\end{array}\right)
$$

where the real and imaginary parts are taken from (3.20). This spinor can be written as a sum of the particle and antiparticle components and reads

$$
\psi(\mathbf{x}, t)=\psi_{\mathrm{P}}(\mathbf{x}, t)+\psi_{\mathrm{A}}(\mathbf{x}, t) .
$$

These two contributions can be expressed, in terms of the complex four-component polarization spinors to be defined below, separately as follows:

$$
\begin{aligned}
& \psi_{\mathrm{P}}(\mathbf{x}, t)=\exp (-\mathrm{i} E t+\mathrm{i} \mathbf{p} \cdot \mathbf{x}) a(\mathbf{p}) \tilde{\alpha}(\mathbf{p})+\exp (+\mathrm{i} E t-\mathrm{i} \mathbf{p} \cdot \mathbf{x}) a^{*}(\mathbf{p}) \tilde{\alpha}^{*}(\mathbf{p}) \\
& \psi_{\mathrm{A}}(\mathbf{x}, t)=\exp (-\mathrm{i} E t+\mathrm{i} \mathbf{p} \cdot \mathbf{x}) b(\mathbf{p}) \tilde{\beta}(\mathbf{p})+\exp (+\mathrm{i} E t-\mathrm{i} \mathbf{p} \cdot \mathbf{x}) b^{*}(\mathbf{p}) \tilde{\beta}^{*}(\mathbf{p})
\end{aligned}
$$

Apparently, $\psi_{\mathrm{P}, \mathrm{A}}=\psi_{\mathrm{P}, \mathrm{A}^{\prime}}^{*}$ and thus the wave functions are real. The polarization spinors $\widetilde{\alpha}$ and $\tilde{\beta}$ can be constructed from the two-component eigenfunctions, given in (3.9), of the helicity operator (3.8). Thus they can be concisely written as

$$
\begin{aligned}
& \tilde{\alpha}(\mathbf{p})=\frac{1}{2}\left(\begin{array}{l}
\left(\varepsilon_{+}(p) u_{+}(\widehat{\mathbf{p}})-\varepsilon_{-}(p) u_{-}^{*}(\widehat{\mathbf{p}})\right)(1-\mathrm{i}) \\
\left(\varepsilon_{+}(p) u_{+}(\widehat{\mathbf{p}})+\varepsilon_{-}(p) u_{-}^{*}(\widehat{\mathbf{p}})\right)(1+\mathrm{i})
\end{array}\right), \\
& \tilde{\beta}(\mathbf{p})=\frac{1}{2}\left(\begin{array}{l}
\left(\varepsilon_{-}(p) u_{-}(\widehat{\mathbf{p}})+\varepsilon_{+}(p) u_{+}^{*}(\widehat{\mathbf{p}})\right)(1-\mathrm{i}) \\
\left(\varepsilon_{-}(p) u_{-}(\widehat{\mathbf{p}})-\varepsilon_{+}(p) u_{+}^{*}(\widehat{\mathbf{p}})\right)(1+\mathrm{i})
\end{array}\right) .
\end{aligned}
$$

Use has again been made of the symbols $\varepsilon_{ \pm}$as defined in (3.16). Using their properties and the orthogonality of $u_{ \pm}$, one can readily show that $\tilde{\alpha}^{\dagger} \widetilde{\alpha}=1$ and $\widetilde{\beta}^{\dagger} \widetilde{\beta}=1$, respectively, $\widetilde{\alpha}^{\dagger} \widetilde{\beta}=0=\widetilde{\beta}^{\dagger} \widetilde{\alpha}$.

We may now insert the spinors (5.3) and (5.4) into the real Majorana equation (2.21) or complex Dirac equation (2.22), to validate that they are solutions of those equations. With the covariant four-momentum $p_{\mu}=(E,-\mathbf{p})$, one finds that the real and imaginary parts of $\tilde{\alpha}=\tilde{\alpha}_{R}+i \tilde{\alpha}_{I}($ resp., $\tilde{\beta})$ must obey the coupled equations:

$$
\bar{\gamma}^{\mu} p_{\mu} \tilde{\alpha}_{\mathrm{I}}+m \tilde{\alpha}_{\mathrm{R}}=0
$$

and similarly

$$
\bar{\gamma}^{\mu} p_{\mu} \tilde{\alpha}_{R}-m \tilde{\alpha}_{I}=0
$$

which can be combined to yield the complex solution of the Dirac equation as

$$
\gamma^{\mu} p_{\mu} \tilde{\alpha}=m \tilde{\alpha}
$$


The same equation must hold true for the polarization spinor $\tilde{\beta}$, and this is indeed the case. The latter equation can explicitly be written in matrix form as $\mathbb{M} \widetilde{\alpha}=0$, with the non-Hermitian matrix

$$
\mathbb{M}=\left(\begin{array}{cccc}
-p_{\mathrm{y}}+\mathrm{i} m & 0 & p_{\mathrm{x}} & -E-p_{\mathrm{z}} \\
0 & -p_{\mathrm{y}}+\mathrm{i} m & E-p_{\mathrm{z}} & -p_{\mathrm{x}} \\
p_{\mathrm{x}} & -E-p_{\mathrm{z}} & p_{\mathrm{y}}+\mathrm{i} m & 0 \\
E-p_{\mathrm{z}} & -p_{\mathrm{x}} & 0 & p_{\mathrm{y}}+\mathrm{i} m
\end{array}\right)
$$

which is the $4 \times 4$-matrix analogue of the Majorana operator $\mathcal{M}$ in (3.7). The validation of (5.9) requires some lengthy algebraic calculations that shall not be done here. Nontrivial solutions of $\widetilde{\alpha}$ to exist require that the determinant of $\mathbb{M}$ vanishes. The corresponding polynomial in the real variable $E$ is of fourth order, yet only yields the two real roots already quoted in (3.4), corresponding to particles and antiparticles.

For the sake of completeness we finally give the full four-component polarization spinor $\alpha$, which in terms of the angles of the momentum unit vector $\widehat{\mathbf{p}}=$ $(\sin \theta \cos \phi, \sin \theta \sin \phi, \cos \theta)$ and $p$ reads as follows:

$$
\tilde{\alpha}(\mathbf{p})=\frac{1}{2}\left(\begin{array}{l}
\left(\varepsilon_{+}(p) \cos \frac{\theta}{2} \mathrm{e}^{-(\mathrm{i} / 2) \phi}+\varepsilon_{-}(p) \sin \frac{\theta}{2} \mathrm{e}^{+(\mathrm{i} / 2) \phi}\right)(1-\mathrm{i}) \\
\left(\varepsilon_{+}(p) \sin \frac{\theta}{2} \mathrm{e}^{+(\mathrm{i} / 2) \phi}-\varepsilon_{-}(p) \cos \frac{\theta}{2} \mathrm{e}^{-(\mathrm{i} / 2) \phi}\right)(1-\mathrm{i}) \\
\left(\varepsilon_{+}(p) \cos \frac{\theta}{2} \mathrm{e}^{-(\mathrm{i} / 2) \phi}-\varepsilon_{-}(p) \sin \frac{\theta}{2} \mathrm{e}^{+(\mathrm{i} / 2) \phi}\right)(1+\mathrm{i}) \\
\left(\varepsilon_{+}(p) \sin \frac{\theta}{2} \mathrm{e}^{+(\mathrm{i} / 2) \phi}+\varepsilon_{-}(p) \cos \frac{\theta}{2} \mathrm{e}^{-(\mathrm{i} / 2) \phi}\right)(1+\mathrm{i})
\end{array}\right) .
$$

Similarly, the polarization spinor $\widetilde{\beta}$ can be derived explicitly from (5.6). This derivation essentially concludes the section on the solution of the real Majorana equation with mass term. We have presented its eigenspinor for particles $\psi_{\mathrm{P}}$ in (5.3) and for antiparticles $\psi_{\mathrm{A}}$ in (5.4). The related quantum fields are readily obtained by interpreting $a$ as annihilation operator, and by replacing $a^{*}$ by $a^{\dagger}$ and interpreting it as the particle creation operator both together obeying the usual fermion anticommutation rule (4.1). The resulting field operator $\Psi_{\mathrm{P}}$ is not Hermitian, though, as $\tilde{\alpha}^{\dagger}$ is not equal to $\tilde{\alpha}^{*}$. The same comments apply to the antiparticle quantum field described by the creation operator $b^{\dagger}$ and polarization spinor $\tilde{\beta}^{*}$.

\section{Summary and Conclusions}

The principal goal of this paper was to rederive and discuss the two-component and four-component Majorana equations completely on their own rather than as a spinoff of the chiral Dirac equation. We obtained these equations including a mass term in a new way by a direct linearization of the relativistic dispersion relation of a massive particle. Thereby we only made use of the complex conjugation operator and the Pauli spin matrices, corresponding to the irreducible representation of the Lorentz group. We then calculated the eigenfunctions of the complex two-component Majorana equation and its related quantum field in a transparent way, exploiting the spin-flip or related chirality 
conjugation operator. Subsequently, we showed the four-component Dirac equation in its real Majorana representation to be the natural outcome of the genuine, two-component, and complex Majorana equation (2.7).

As the analysis of this version of the Majorana equation clearly reveals, the two associated Majorana particles (with creation operator $a^{\dagger}$ and similarly $b^{\dagger}$ ) represent linearly independent states and are not their own antiparticles, when being defined like in reference [7] in the sense of having positive or negative energies (3.4). We recall here that the original eigenfunctions (3.19) and (3.20) result from a superposition of different states and thus do neither describe a pure helicity state nor do they have a well-defined four-momentum. These distinct features only emerge by evaluating the expectation values (4.16) and (4.18) of the quantum fields. They do in fact describe particles and antiparticles with opposite helicities, consistently with the standard perception of neutrinos and antineutrinos.

The symmetries of the two-component Majorana equation were also analysed. It was shown to obey time reversal and parity, yet apparently violates chirality conjugation by construction. As the Majorana quantum field is uncharged, the common term "charge conjugation" does not appear adequate here, but better Lorentz-covariant complex conjugation [8]. Even more appropriately, while stressing clearly the physical meaning, one may speak of "chirality conjugation," which refers to the two conjugate versions of the Majorana equation having opposite chirality. So, the Majorana equation (2.7), while breaking chiral symmetry by definition, is as basic as Dirac's equation and when considered as a quantum field can describe massive and uncharged right-handed or left-handed particles or antiparticles, corresponding to massive neutrinos. In our opinion, the approach starting from scratch with (2.5) gives us new insights into the nature of the Majorana equation and shows that it deserves to be considered in its own right.

\section{References}

[1] E. Marsch, "The two-component Majorana equation-novel derivations and known symmetries," Journal of Modern Physics, vol. 2, pp. 1109-1114, 2011.

[2] P. A. M. Dirac, "The quantum theory of the electron," Proceedings of the Royal Society London, vol. 117, pp. 610-624, 1928.

[3] E. Majorana, “Teoria simmetrica dell' elettrone e del positrone," Nuovo Cimento, vol. 14, pp. 171-184, 1937.

[4] R. N. Mohapatra and P. B. Pal, Massive Neutrinos in Physics and Astrophysics, World Scientific, Singapore, 2004.

[5] M. Fukugita and T. Yanagida, Physics of Neutrinos and Applications to Astrophysics, Springer, Berlin, Germany, 2003.

[6] K. M. Case, "Reformulation of the Majorana theory of the neutrino," Physical Review, vol. 107, pp. 307-316, 1957.

[7] P. D. Mannheim, "Introduction to Majorana masses," International Journal of Theoretical Physics, vol. 23, no. 7, pp. 643-674, 1984.

[8] P. B. Pal, "Dirac, Majorana, and Weyl fermions," American Journal of Physics, vol. 79, pp. 485-498, 2011.

[9] A. Das, Lectures on Quantum Field Theory, World Scientific Publishing, Singapore, 2008.

[10] M. Kaku, Quantum Field Theory. A Modern Introduction, The Clarendon Press Oxford University Press, New York, NY, USA, 1993.

[11] W. Pauli, "Zur quantenmechanik des magnetischen elektrons," Zeitschrift fur Physik A, vol. 43, pp. 601-623, 1927.

[12] H. Jehle, "Two-component wave equations," Physical Review, vol. 75, p. 1609, 1949.

[13] H. Weyl, "Elektron und graviton I," Zeitschrift fur Physik, vol. 56, pp. 330-352, 1929. 


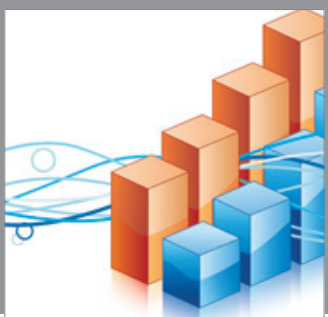

Advances in

Operations Research

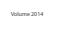

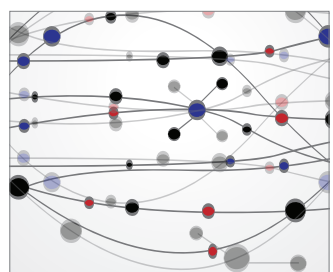

\section{The Scientific} World Journal
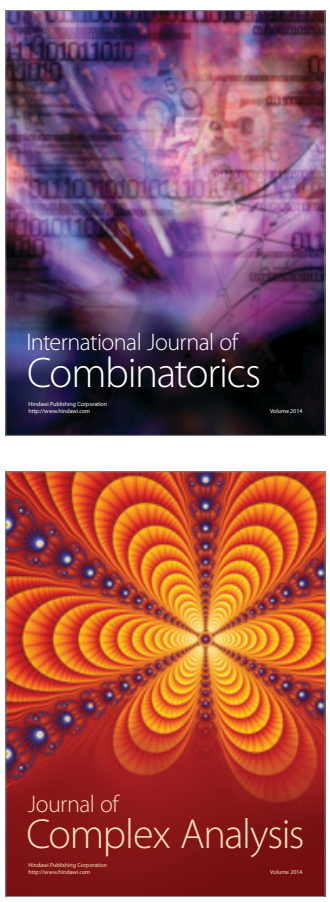

International Journal of

Mathematics and

Mathematical

Sciences
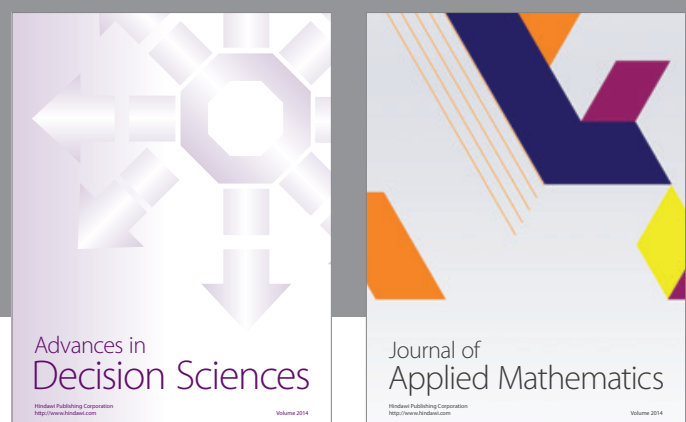

Journal of

Applied Mathematics
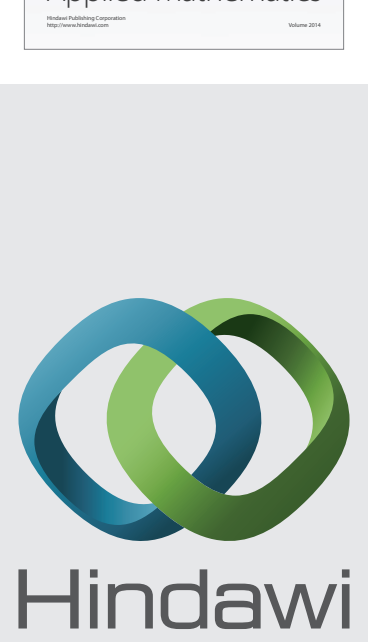

Submit your manuscripts at http://www.hindawi.com
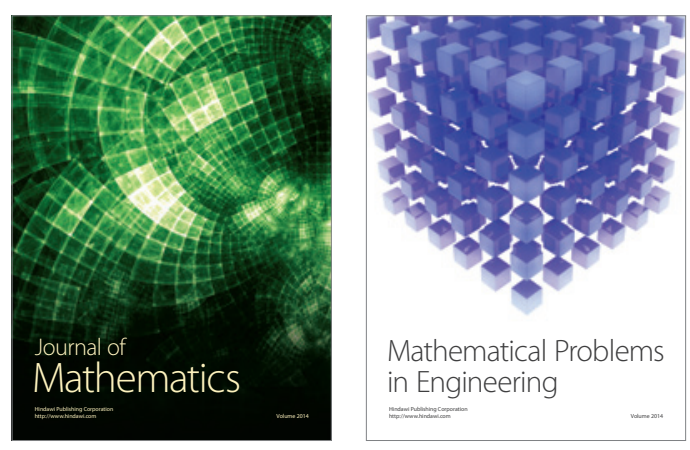

Mathematical Problems in Engineering
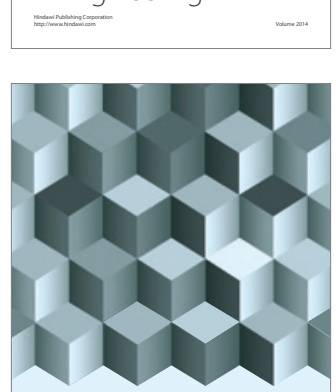

Journal of

Function Spaces
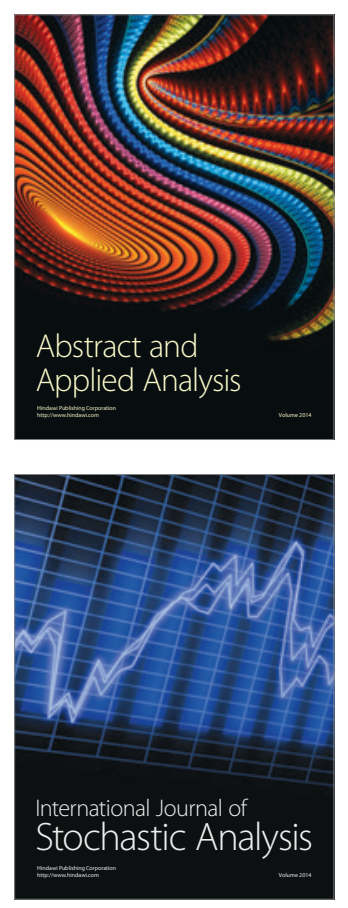

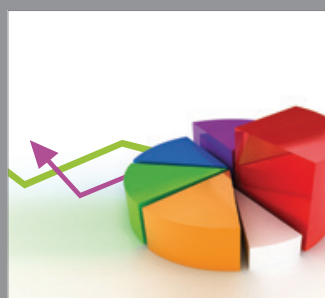

ournal of

Probability and Statistics

Promensencen
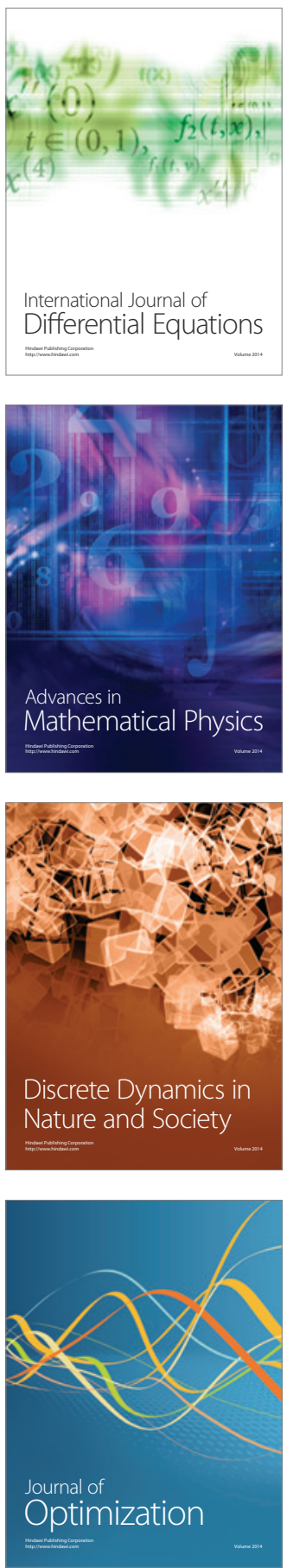